\title{
Subthreshold membrane potential dynamics of posterior parietal cortical neurons coupled with hippocampal ripples
}

\author{
Y. SATO ${ }^{1}$, H. MIZUNO ${ }^{1}$, N. MATSUMOTO ${ }^{1 *}$ (1) and Y. IKEGAYA ${ }^{1,2,3}$ \\ ${ }^{1}$ Graduate School of Pharmaceutical Sciences, The University of Tokyo, Tokyo 113-0033, Japan \\ ${ }^{2}$ Institute for AI and Beyond, The University of Tokyo, Tokyo 113-0033, Japan \\ ${ }^{3}$ Center for Information and Neural Networks, National Institute of Information and Communications \\ Technology, Suita City, Osaka, 565-0871, Japan
}

Received: March 6, 2020 • Accepted: November 17, 2020

Published online: March 25, 2021

(c) 2021 The Author(s)

\begin{abstract}
During behavioral states of immobility, sleep, and anesthesia, the hippocampus generates high-frequency oscillations called ripples. Ripples occur simultaneously with synchronous neuronal activity in the neocortex, known as slow waves, and contribute to memory consolidation. During these ripples, various neocortical regions exhibit modulations in spike rates and local field activity irrespective of whether they receive direct synaptic inputs from the hippocampus. However, little is known about the subthreshold dynamics of the membrane potentials of neocortical neurons during ripples. We patch-clamped layer $2 / 3$ pyramidal cells in the posterior parietal cortex (PPC), a neocortical region that is involved in allocentric spatial representation of behavioral exploration and sequential series of relevant action potentials during ripples. We simultaneously monitored the membrane potentials of post hoc-identified PPC neurons and the local field potentials of the hippocampus in anesthetized mice. More than $50 \%$ of the recorded PPC neurons exhibited significant depolarizations and/or hyperpolarizations during ripples. Histological inspections of the recorded neurons revealed that the ripple-modulated PPC neurons were distributed in the PPC in a spatially non-biased fashion. These results suggest that hippocampal ripples are widely but selectively associated with the subthreshold dynamics of the membrane potentials of PPC neurons even though there is no monosynaptic connectivity between the hippocampus and the PPC.
\end{abstract}

\footnotetext{
* Corresponding author. Laboratory of Chemical Pharmacology, Graduate School of Pharmaceutical Sciences, The University of Tokyo, Japan 7-3-1 Hongo, Bunkyo-ku, Tokyo 113-0033, Japan. Tel.: +81 35841 4781; fax: +8135841 4786. E-mail: nobuyoshi@matsumoto.ac
} 


\section{KEYWORDS}

hippocampus, membrane potential, whole-cell recording, posterior parietal cortex, ripple

\section{INTRODUCTION}

Cortico-hippocampal interactions, particularly during sleep and quiet wakefulness, are instrumental in memory consolidation [1,2]. During behavioral states or under anesthesia, spontaneous activity in the neocortex is modulated by slow $(0.5-4 \mathrm{~Hz})$ oscillations [3], which are dominant during non-rapid eye movement sleep. On the other hand, hippocampal activity during non-rapid eye movement sleep is dominated by ripples, high-frequency oscillations that contribute to memory consolidation $[4,5]$. Hippocampal neurons are sequentially reactivated during ripples in a more time-compressed manner than the temporally patterned sequences of neuronal spikes that occur during active exploration before sleep [6-8]. Compressed spike sequences are also observed in the posterior parietal cortex (PPC) [9], in which neuronal activity is modulated during hippocampal ripples $[10,11]$. Moreover, magnetic resonance imaging (MRI) and voltage-sensitive dye imaging $[12,13]$ have demonstrated that PPC activity is increased immediately after hippocampal ripples. However, the relationship between hippocampal ripples and the subthreshold dynamics of membrane potentials of PPC neurons has received little attention.

Although there are no direct synaptic interactions between the hippocampus and the PPC, studies using artificial electrical stimulation have revealed a functional relationship between these regions [14-16]. However, the intrinsic interrelations of spontaneous neuronal activity under more physiological conditions are not yet well understood. We hypothesize that spontaneously occurring hippocampal ripples exert a functional impact on PPC neuronal activity and that this interaction is reflected in the subthreshold dynamics of the membrane potentials of PPC neurons. We simultaneously recorded the membrane potentials of layer $2 / 3$ pyramidal cells in the PPC and local field potentials (LFPs) from the hippocampal CA1 stratum pyramidale using in vivo wholecell patch-clamp recording techniques and extracellular field recording techniques, respectively.

\section{MATERIALS AND METHODS}

\section{Animal ethics}

ICR mice (Japan SLC, Shizuoka, Japan) were used in these experiments. The animal experiments were performed with the approval of the Animal Experiment Ethics Committee of the University of Tokyo (approval number: P29-9) and according to the University of Tokyo guidelines for the care and use of laboratory animals. These experimental protocols were carried out in accordance with the Fundamental Guidelines for Proper Conduct of Animal Experiment and Related Activities in Academic Research Institutions (Ministry of Education, Culture, Sports, Science and Technology, Notice No. 71 of 2006), the Standards for Breeding and Housing of and Pain Alleviation for Experimental Animals (Ministry of the Environment, Notice No. 88 of 2006) and the Guidelines on the Method of Animal Disposal (Prime Minister's Office, Notice No. 40 of 1995). All animals were housed under a 12-h dark-light cycle (lights on from 7:00 to 19:00) at $22 \pm 1{ }^{\circ} \mathrm{C}$ with ad libitum access to food and water. 


\section{Surgery}

Whole-cell recordings were obtained from postnatal 28- to 40-day-old male ICR mice as previously described [17-19]. The mice were anesthetized with intraperitoneal urethane $(2.25 \mathrm{~g} / \mathrm{kg})$. Anesthesia was confirmed by the absence of the paw withdrawal, whisker movement, and eyeblink reflexes. The skin was subsequently removed from the head, and the animal was placed on a stereotaxic apparatus (SR-6M-HT, Narishige, Tokyo, Japan). A craniotomy $(2.5 \times 2.0$ $\mathrm{mm}^{2}$ ) was performed above the right hemisphere centered $2.0 \mathrm{~mm}$ posterior to bregma and 2.5 $\mathrm{mm}$ ventrolateral to the sagittal suture, and the dura was surgically removed. The exposed cortical window was covered with $1.7 \%$ agar at a thickness of $1.5 \mathrm{~mm}$.

\section{Electrophysiology}

Whole-cell recordings were obtained from neurons in the PPC (AP: $1.2-3.5 \mathrm{~mm}$ posterior to bregma; ML: $0.60-2.51 \mathrm{~mm}$ from the sagittal suture; DV: $40-520 \mu \mathrm{m}$ ventral to the dura) or the retrosplenial cortex (RSC; AP: $1.5-3.2 \mathrm{~mm}$ posterior to bregma; $\mathrm{ML}: 0.44-0.60 \mathrm{~mm}$ from the sagittal suture; DV: 170-630 $\mu \mathrm{m}$ ventral to the dura) using borosilicate glass electrodes (4-7 $\mathrm{M} \Omega$ ). Principal cells were identified on the basis of their regular spiking properties and by post hoc histological analysis. For current-clamp recordings, the intrapipette solution consisted of the following reagents (in $\mathrm{mM}$ ): $135 \mathrm{~K}$-gluconate, $4 \mathrm{KCl}, 10 \mathrm{HEPES}, 10$ creatine phosphate, 4 MgATP, $0.3 \mathrm{Na}_{2} \mathrm{GTP}, 0.3$ EGTA ( $\mathrm{pH} 7.3$ ), and $0.2 \%$ biocytin. The recorded membrane potentials were corrected offline by compensating liquid junction potentials as described previously [19]; hereafter, the corrected membrane potentials were analyzed. At the beginning of each experiment, we injected 500-ms rectangular currents of $-200-200 \mathrm{pA}$ into the cell in 50-pA ascending steps to examine whether it was a putative excitatory or inhibitory neuron based on its spike responses; note that in general, excitatory and inhibitory neurons exhibit regular-spiking and fast-spiking firing patterns, respectively. Regular-spiking neurons were selected for the subsequent analyses. Cells were discarded when the series resistance exceeded $75 \mathrm{M} \Omega$ or the mean of the corrected membrane potentials exceeded $-55 \mathrm{mV}$. Moreover, the recordings were truncated when the resting potential increased by more than $8 \mathrm{mV}$ from its value at the onset of the recording. LFPs were obtained from the CA1 stratum pyramidale using a tungsten electrode (UEWMGCSEKNNM, FHC, Bowdoin, ME, USA) coated with DiI. The location of the tungsten electrode was detected by post hoc observation of the DiI track. The signals from the glass electrodes were amplified using a MultiClamp 700B amplifier, the signals from the tungsten electrode were amplified using a DAM80 AC differential amplifier, and all signals were digitized at a sampling rate of $20 \mathrm{kHz}$ using either a Digidata $1320 \mathrm{~A}$ or $1440 \mathrm{~A}$ digitizer that was controlled by pCLAMP 10.7 software (Molecular Devices, San Jose, CA, USA). The data were analyzed offline using custom-made MATLAB (R2018b, Natick, MA, USA) routines.

\section{Histology}

Following each experiment, the electrode was carefully removed from the brain. To visualize the patch-clamped neurons, the mice were transcardially perfused with $4 \%$ paraformaldehyde, and the brains were fixed in $4 \%$ paraformaldehyde overnight. We then recovered the morphology of the recorded neurons by two following methods. The method was randomly chosen regardless of neuronal firing pattern, the age of the mouse, or other recording conditions. (i) The brains 
were coronally or sagittally sectioned at a thickness of $100 \mu \mathrm{m}$ using a vibratome. The sections were incubated with $2 \mu \mathrm{g} / \mathrm{ml}$ streptavidin-Alexa Fluor 594 conjugate and $0.2 \%$ Triton X-100 for $4 \mathrm{~h}$, followed by incubation with $0.1 \%$ Hoechst 33342 (H1399, Thermo Fisher Scientific, Waltham, MA, USA) or 0.4\% NeuroTrace 435/455 (N21479, Thermo Fisher Scientific, Waltham, MA, USA) for $4 \mathrm{~h}$. (ii) For a separate preparation, brains from a different subset were coronally sectioned at a thickness of $500 \mu \mathrm{m}$ using a brain slicer (MBS-S0.5C, Brain Science Idea, Osaka, Japan). These sections were fixed in $10 \%$ paraformaldehyde with $0.1 \%$ Hoechst 33342 at $4{ }^{\circ} \mathrm{C}$ for $30 \mathrm{~min}$. After the solution was rinsed off, the sections were incubated in IsoScaleSQ with $2 \mu \mathrm{g} /$

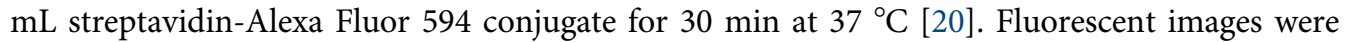
acquired using a confocal microscope (FV1200, Olympus, Tokyo, Japan) and were subsequently merged.

\section{Hippocampal ripple detection}

To detect hippocampal ripple events, hippocampal LFP traces were bandpass filtered at 150-250 $\mathrm{Hz}$, and the root-mean-square (RMS) power was calculated in the band with a bin size of $20 \mathrm{~ms}$. The threshold for ripple detection was set to $4 \times$ standard deviations (SDs) above the mean. The onsets and offsets of the ripples were marked at the points when the ripple power first exceeded and dropped below $4 \times$ SDs above the mean, respectively, and events with a duration of $<20 \mathrm{~ms}$ were excluded.

\section{Determination of action potential thresholds}

The threshold of each action potential was set as the membrane potential value at which the time differential of the membrane potential crossed $10 \mathrm{~V} / \mathrm{s}$ [21]. Then, among all the thresholds, we defined the top $90 \%$ value as the action potential threshold of each neuron.

\section{Definition of subthreshold membrane potential activity}

The amplitudes of the subthreshold fluctuations of PPC neurons triggered by ripples were detected within 0 to $+100 \mathrm{~ms}$ from the ripple onset. For all ripple events, the maximum absolute changes in membrane potential were detected as the polarizations $\left(\Delta V_{m}\right)$, and the baseline $\Delta V_{m}$ was determined as the mean of the membrane potentials within -20 to $0 \mathrm{~ms}$ from the ripple onset. When the relative value of $\Delta V_{m}$ was positive or negative, the event was defined as depolarization (i.e., the Up state) or hyperpolarization (i.e., the Down state), respectively. If the $\Delta V_{m}$ was zero, the event was excluded from the analysis because of the zero-division problem.

\section{Statistical analysis}

Data analyses were performed using MATLAB. For comparison of the cumulative probability of $\Delta V_{m}$ being locked to the ripple onset and to times other than the ripple onset, the KolmogorovSmirnov test was used. $P<0.05$ was considered statistically significant. A given neuron was defined as non-responsive when its $\Delta V_{m}$ was not significant. For comparison of the ratio of cell types in the PPC and the RSC, the chi-squared test was used. To investigate correlations between depolarization or hyperpolarization of PPC neurons and the age of the animal, the $t$-test of no correlation was used. 


\section{RESULTS}

We obtained whole-cell patch-clamp recordings from PPC layer $2 / 3$ neurons and extracellular recordings of LFPs from the hippocampal CA1 stratum pyramidale of urethane-anesthetized mice and investigated the $\Delta V_{m}$ dynamics of PPC neurons and hippocampal ripples (Fig. 1).

We found that the subthreshold $\Delta V_{m}$ responses immediately after the ripple onset varied among cells and roughly identified three types of PPC neurons: (i) depolarizing cells (Fig. 2AC), (ii) non-responsive cells (i.e., neither significantly depolarizing nor hyperpolarizing; see Materials and methods) (Fig. 2D-F), and (iii) hyperpolarizing cells (Fig. 2G-I). More specifically, for each PPC neuron, we plotted the cumulative distributions of $\Delta V_{m}$ for all recorded ripple events by separating cases of $\Delta V_{m}>0$ (depolarization) and $\Delta V_{m}<0$ (hyperpolarization); note that $\Delta V_{m}$ varied from ripple event to ripple event even within the same cell. For each cell, we compared the overall $\Delta V_{m}$ distributions to their surrogates, i.e., the baseline $\Delta V_{m}$ distributions obtained across all recording periods without ripple events (see Materials and methods), and detected significant differences between the real and surrogate datasets using the Kolmogorov-Smirnov test. These ripple-associated depolarizations and hyperpolarizations in PPC neurons were likely observed at Down-to- $U$ p and Up-to-Down transitions, respectively (Fig. 2A, D, G). Of a total of 24 PPC neurons from 21 mice, $13(54 \%)$ cells exhibited significant $\Delta V_{m}$ changes $(P<0.05$; Table 1$)$. Of these 13 PPC neurons, three neurons were significantly depolarized (Fig. 3A, red and filled), and seven were significantly hyperpolarized (Fig. 3A, blue and filled). The remaining three neurons exhibited both significant depolarizations and hyperpolarizations as a whole (Fig. 3A, green and filled); that is, they tended to respond to every ripple event with either depolarization or hyperpolarization. Neither depolarization nor hyperpolarization of PPC neurons was significantly correlated with the age of the animal $\left(P=0.86, t_{11}=\right.$ $0.18, R^{2}=2.98 \times 10^{-3}$ (depolarization), $n=13$ neurons, $t$-test of no correlation; $P=0.16, t_{11}=$ $1.53, R^{2}=0.17$ (hyperpolarization), $n=13$ neurons, $t$-test of no correlation).
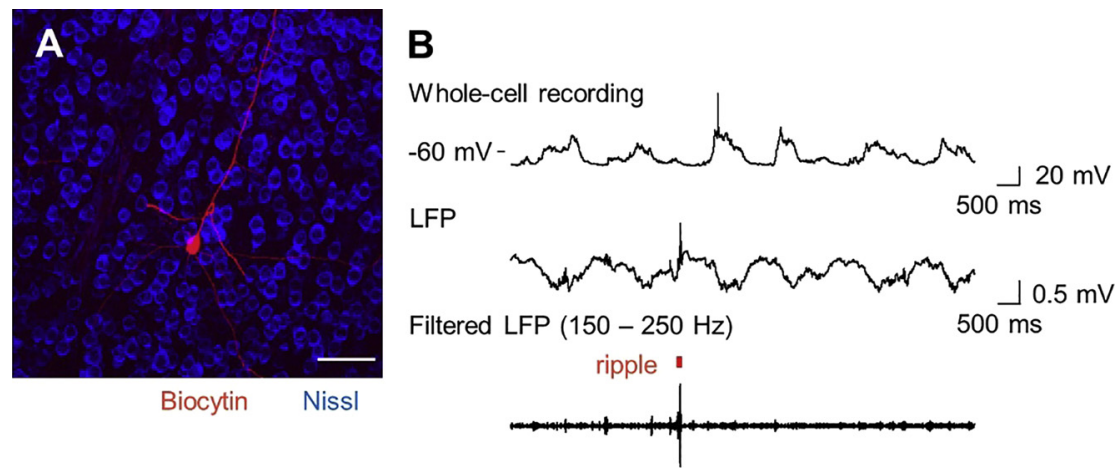

Fig. 1. Simultaneous recordings of the membrane potentials of PPC layer $2 / 3$ pyramidal cells and hippocampal LFPs. (A) A representative image of a biocytin-visualized whole-cell recorded neuron (red) in Nissl counterstained section (blue). (B) Top: A representative membrane potential trace recorded from a PPC neuron. Middle: A representative LFP trace recorded from the hippocampus. Membrane potential and LFP traces were simultaneously recorded. Bottom: The LFP trace after 150 -to $250-\mathrm{Hz}$ filtering. The red line indicates the onset of a hippocampal ripple 

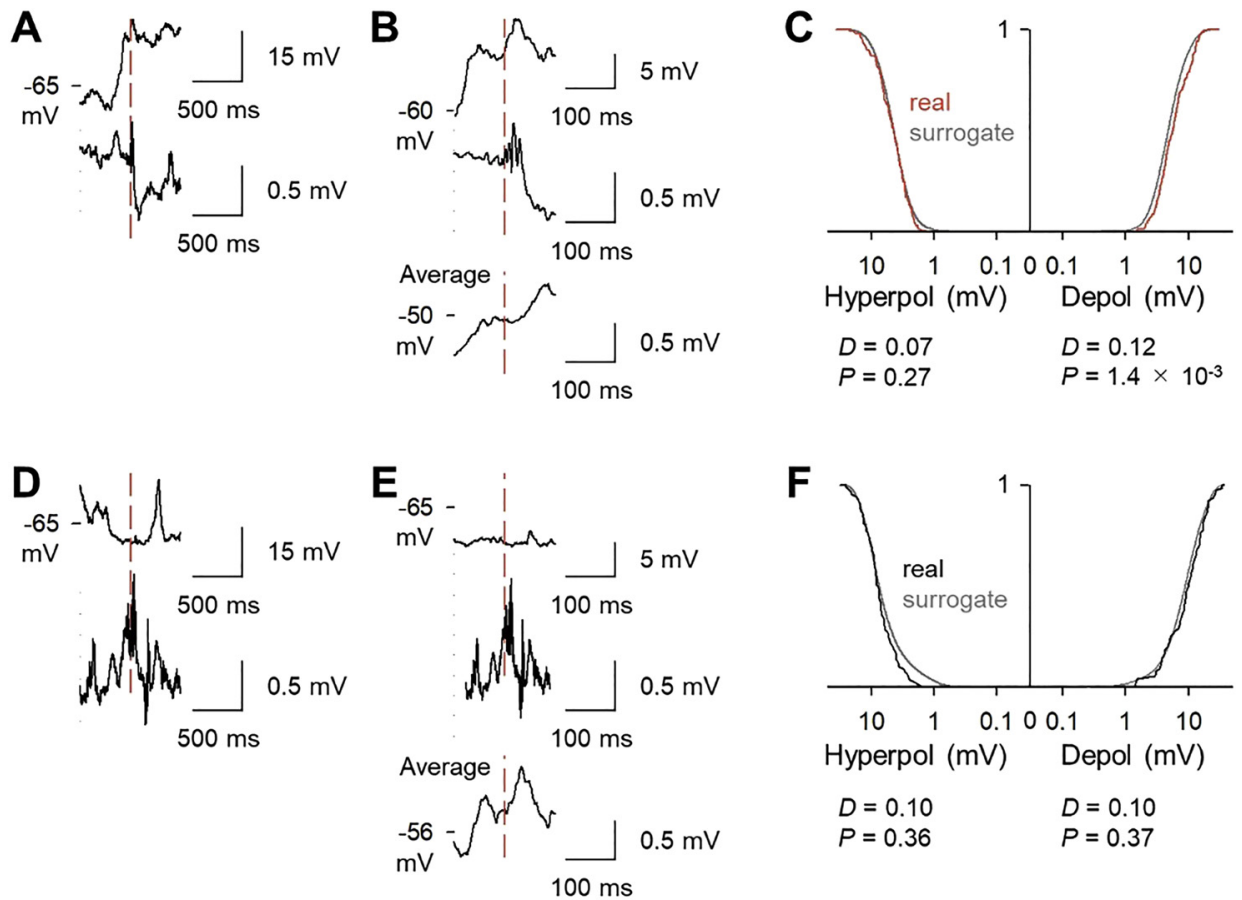

Hyperpol $(\mathrm{mV})$ Depol $(\mathrm{mV})$

$$
\begin{array}{ll}
D=0.10 & D=0.10 \\
P=0.36 & P=0.37
\end{array}
$$
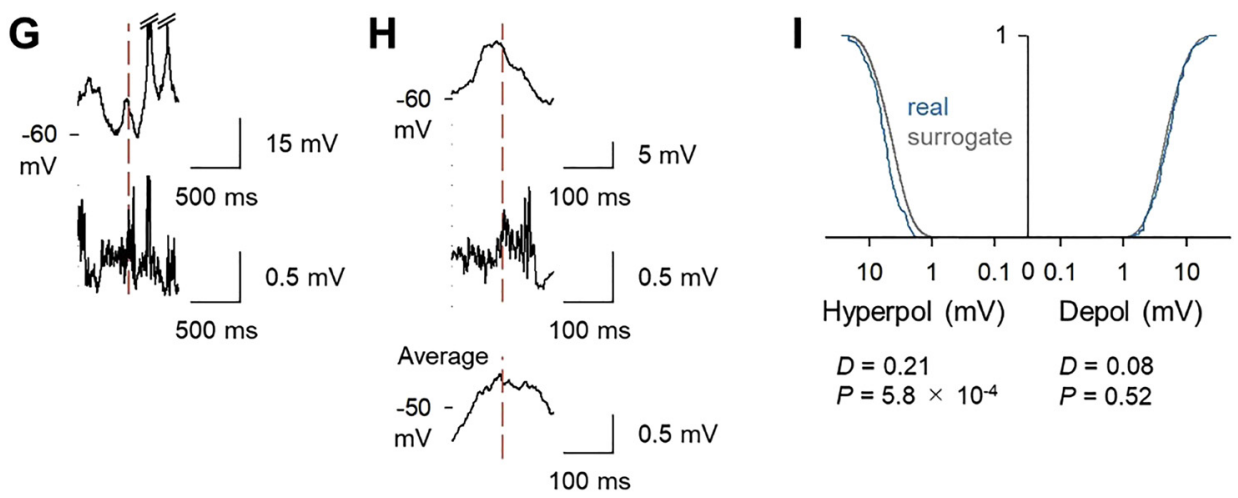

Hyperpol $(\mathrm{mV}) \quad$ Depol $(\mathrm{mV})$

$$
\begin{array}{ll}
D=0.21 & D=0.08 \\
P=5.8 \times 10^{-4} & P=0.52
\end{array}
$$

Fig. 2. Different patterns of the $\Delta V_{m}$ dynamics of PPC neurons during hippocampal ripples. (A) Representative membrane potential fluctuations of PPC neurons (top) exhibiting depolarization during the simultaneous recording of LFP traces (including ripples) from the hippocampus (bottom). Red indicates the onset of hippocampal ripples. (B) Magnified traces of (A) (top, middle) and the average membrane potential fluctuations of PPC neurons (bottom). (C) Cumulative probabilities of $\Delta V_{m}$ of PPC neurons at ripple event times (real) and at times without ripples (surrogate). The $D$ and $P$ values were calculated by the Kolmogorov-Smirnov test. (D-F) The same as (A-C) but for PPC neurons not exhibiting apparent responses. (G-I) The same as $(\mathrm{A}-\mathrm{C})$ but for PPC neurons exhibiting hyperpolarization 
Table 1. Statistics of all recorded neurons

\begin{tabular}{|c|c|c|c|c|c|}
\hline \multirow[b]{2}{*}{ PPC Neuron ID } & \multicolumn{2}{|c|}{ Hyperpolarization } & \multicolumn{2}{|c|}{ Depolarization } & \multirow[b]{2}{*}{ Classification } \\
\hline & $P$ value & $D$ value & $P$ value & $D$ value & \\
\hline 1 & 0.0670 & 0.1883 & 0.0162 & 0.1923 & Depolarization only \\
\hline 2 & 0.2824 & 0.1611 & 0.0318 & 0.2799 & \\
\hline 3 & 0.2653 & 0.0668 & 0.0013 & 0.1197 & \\
\hline 4 & 0.0044 & 0.1850 & 0.1828 & 0.1244 & Hyperpolarization only \\
\hline 5 & 0.0006 & 0.2054 & 0.5185 & 0.0830 & \\
\hline 6 & 0.0036 & 0.1623 & 0.3878 & 0.1041 & \\
\hline 7 & 0.0319 & 0.2079 & 0.3417 & 0.1357 & \\
\hline 8 & 0.0159 & 0.0901 & 0.7720 & 0.0379 & \\
\hline 9 & 0.0004 & 0.1534 & 0.5197 & 0.0668 & \\
\hline 10 & 0.0040 & 0.1813 & 0.2495 & 0.1103 & \\
\hline 11 & 0.0001 & 0.2521 & 0.0032 & 0.2295 & Both \\
\hline 12 & 0.0404 & 0.1558 & 0.0096 & 0.1567 & \\
\hline 13 & 0.0000 & 0.1989 & 0.0111 & 0.1177 & \\
\hline 14 & 0.7163 & 0.0987 & 0.7380 & 0.0822 & Neither \\
\hline 15 & 0.3603 & 0.1006 & 0.3652 & 0.1040 & \\
\hline 16 & 0.2328 & 0.1498 & 0.0523 & 0.1788 & \\
\hline 17 & 0.5009 & 0.1608 & 0.6863 & 0.1233 & \\
\hline 18 & 0.0582 & 0.2952 & 0.5610 & 0.1536 & \\
\hline 19 & 0.5333 & 0.1511 & 0.5024 & 0.1576 & \\
\hline 20 & 0.2839 & 0.0773 & 0.0920 & 0.0909 & \\
\hline 21 & 0.6164 & 0.0671 & 0.6614 & 0.0664 & \\
\hline 22 & 0.8002 & 0.0643 & 0.7923 & 0.0725 & \\
\hline 23 & 0.7078 & 0.1160 & 0.6929 & 0.1191 & \\
\hline \multirow[t]{2}{*}{24} & 0.1483 & 0.1583 & 0.7231 & 0.1099 & \\
\hline & \multicolumn{2}{|c|}{ Hyperpolarization } & \multicolumn{2}{|c|}{ Depolarization } & \\
\hline RSC Neuron ID & $P$ value & $D$ value & $P$ value & $D$ value & Classification \\
\hline 1 & 0.1885 & 0.1934 & 0.0165 & 0.1935 & Depolarization only \\
\hline 2 & 0.0199 & 0.1223 & 0.5499 & 0.0737 & Hyperpolarization only \\
\hline 3 & 0.0008 & 0.1752 & 0.7832 & 0.0671 & \\
\hline 4 & 0.0000 & 0.2487 & 0.0507 & 0.1503 & \\
\hline 5 & 0.0444 & 0.1995 & 0.3287 & 0.1268 & \\
\hline 6 & 0.0022 & 0.2403 & 0.0026 & 0.2240 & Both \\
\hline 7 & 0.1063 & 0.1681 & 0.6145 & 0.0986 & Neither \\
\hline 8 & 0.4997 & 0.1116 & 0.3961 & 0.1326 & \\
\hline
\end{tabular}

$P$ and $D$ values are calculated by the Kolmogorov-Smirnov test.

Since the RSC is one of the candidate regions for hippocampus-to-PPC propagation [22], we further obtained whole-cell patch-clamp recordings from RSC layer $2 / 3$ neurons and extracellular recordings of LFPs from the hippocampal CA1 stratum pyramidale (Fig. 3). Of a total of eight RSC neurons from seven mice, six (75\%) cells exhibited significant $\Delta V_{m}$ changes. Of these six RSC neurons, one neuron was significantly less depolarized, whereas four were significantly 

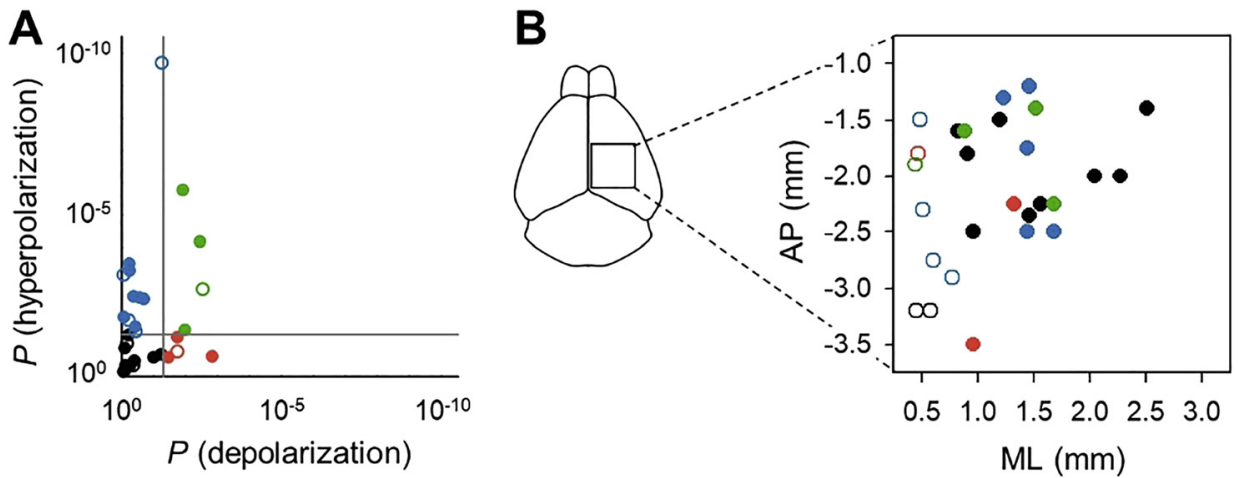

Fig. 3. Classification of $\Delta V_{m}$ patterns of PPC neurons during hippocampal ripples. (A) Distribution of the $P$ values for depolarization (abscissa) and hyperpolarization (ordinate) determined by the KolmogorovSmirnov test. Each dot indicates a single neuron. Blue: Significantly hyperpolarized neurons during ripples. Red: Significantly depolarized neurons. Green: Significantly depolarized and hyperpolarized neurons. Black: Non-responsive neurons. Filled: PPC neurons. Open: RSC neurons. $n=24$ neurons from 21 mice (PPC), $n$ $=8$ neurons from 7 mice (RSC). (B) Top view of the coordinates of visualized recorded neurons in the PPC (filled) and the RSC (open). $n=20$ (PPC) and 8 (RSC) biocytin-identified neurons

hyperpolarized. The remaining neuron exhibited both significant depolarizations and hyperpolarizations (Fig. 3A, open). The ratios of these cell types were not significantly different between the PPC and the RSC $\left(P=0.71, \chi^{2}=1.40\right.$, chi-squared test $)$.

We investigated whether the differences in the $\Delta V_{m}$ responses of individual cells were associated with their anatomical locations in the PPC. We succeeded in visualizing 20 PPC neurons (83\%) after the recordings. Post hoc histological analyses revealed no apparent relationship between spatial distribution and the response type in either the PPC or the RSC (Fig. 3B).

\section{DISCUSSION}

In this study, we simultaneously monitored the membrane potentials of neurons in layer $2 / 3$ of the PPC and hippocampal ripples using a combination of in vivo whole-cell recording and field recording techniques. We demonstrated that more than half of the recorded PPC neurons exhibited temporal $\Delta V_{m}$ modulation that was associated with hippocampal ripple events at subthreshold $\Delta V_{m}$ levels.

Recent studies have proposed that hippocampal ripples during non-rapid eye movement sleep mediate memory consolidation through the reactivation of neocortical neurons $[23,24]$. From the spatiotemporal point of view, a study using MRI demonstrated that hippocampal ripples precede the activation of the neocortex [13]. Moreover, voltage-sensitive dye imaging previously confirmed that the activity of most neocortical regions, including the PPC, is increased during hippocampal ripples [12]. Indeed, neuronal reactivation during hippocampal ripples has been reported in several neocortical regions, including the PPC, even though they receive no direct axonal projections from the hippocampus [25]. For example, a recent study 
showed that while an animal explores a circular platform, PPC neuronal activity exhibits egocentric metrics and thereafter is replayed in a time-compressed manner approximately 100 ms after the onset of hippocampal ripples during post-task sleep [11]. We showed that the $\Delta V_{m}$ of PPC neurons within $100 \mathrm{~ms}$ after ripple onset was different from the baseline $\Delta V_{m}$ during non-ripple periods; note that our data were obtained from urethane-anesthetized mice, but urethane anesthesia at least in part resembles natural sleep [26]. Interestingly, we found not only ripple-locked depolarized neurons but also ripple-locked hyperpolarized neurons and both ripple-locked depolarized and hyperpolarized neurons. In other words, a portion of PPC neurons was either excited or inhibited during ripples. Additionally, some cells were both excited during some ripple events and inhibited during other ripple events. This variety and flexibility in $\Delta V_{m}$ dynamics indicates that a population of PPC neurons is selectively defined to fire spikes during ripples, whereas other populations are suppressed or occasionally fire spikes depending on the ripple event, suggesting that complex coordination in microcircuit-specific excitation generates the spike sequences of selected cell ensembles.

Hippocampal ripples provide allocentric information that is represented by the sequential reactivation of hippocampal place cells, a phenomenon called memory replay [6-8]. Spikes are triggered by large depolarizations that reach the spike threshold during ripples [27]. On the other hand, the PPC encodes egocentric as well as allocentric information $[11,28,29]$, but no studies have described the subthreshold $\Delta V_{m}$ of PPC neurons during ripples. Our study is the first to show that, similar to those of hippocampal pyramidal cells, the depolarizations of a specific population of PPC neurons are time-locked to hippocampal ripples. Intracellular recordings from multiple neurons would confirm whether these ripple-associated depolarizations of PPC neurons underlie the sequential reactivation of ripple-locked spikes in the PPC neuronal population by allowing the calculation of the sequential delay in the latency of the depolarizations.

It remains unknown which brain regions mediate information transfer between the hippocampus and the PPC. One candidate is the entorhinal cortex, which receives synaptic inputs from the hippocampus and sends synaptic outputs to the PPC. More specifically, pyramidal neurons in layer 5 of the medial entorhinal cortex receive monosynaptic excitatory inputs from the hippocampal CA1 region after ripples [30]. Consistent with this phenomenon, the hippocampal-entorhinal-PPC route is believed to be involved in encoding location and navigation [29, 31]. Other candidates include the RSC and the postrhinal cortex, which make synaptic connections with both the entorhinal cortex and the PPC [22, 32]. Aligned to hippocampal ripples, we found the same fraction of depolarized and hyperpolarized neurons between the RSC and the PPC, suggesting that these two regions play similar roles in transmitting neural information from the hippocampus. Moreover, there were fewer depolarized neurons than hyperpolarized neurons in the RSC as well as in the PPC during ripples, which is consistent with a previous report showing that RSC excitatory neurons are inhibited immediately after hippocampal ripples [33]. Simultaneous whole-cell recordings from the PPC and RSC and extracellular field recordings from the hippocampus would provide precise evidence that the RSC mediates hippocampus-to-PPC transmission by allowing analysis of the sequential delay of depolarization in the PPC and RSC relative to the hippocampal ripple onset.

Previous studies have reported that somatosensory cortical myelination is immature until postnatal day 30 [34] and that myelination in the corpus callosum is still in progress on postnatal day $28[35,36]$. Myelination of the primary sensory and motor cortices precedes that 
of associational cortical areas, including the PPC, the hippocampus, the entorhinal cortex, and the RSC [37]. Since the maturation of axonal myelination is involved in the propagation of neural activity, we asked whether the age of animals contributes to changes in membrane potentials. However, the age (i.e., 28-to-40 day old) of the developing animals and membrane potential changes were not significantly correlated. Simultaneous recording of the membrane potentials of PPC neurons and hippocampal ripples from mature animals would reveal a more precise relationship between age-dependent myelination and the propagation of hippocampal ripples.

Because we recorded hippocampal ripples exclusively from anesthetized mice, their activities did not convey spatial information; however, we found different types of PPC neurons in terms of $\Delta V_{m}$ during ripples. Simultaneous patch-clamp recordings from multiple PPC neurons in awake animals will bridge the gap between the diversity in the $\Delta V_{m}$ dynamics of PPC neurons and their physiological function by, for example, illustrating the intracellular mechanisms that determine the PPC cell population that participates in the reactivation of egocentric and allocentric information.

\section{ACKNOWLEDGMENTS}

This work was supported by JST ERATO (JPMJER1801), Institute for AI and Beyond of the University of Tokyo, and JSPS Grants-in-Aid for Scientific Research (18H05525, 20K15926).

\section{REFERENCES}

1. Maingret N, Girardeau G, Todorova R, Goutierre M, Zugaro M. Hippocampo-cortical coupling mediates memory consolidation during sleep. Nat Neurosci 2016; 19: 959-64.

2. Todorova R, Zugaro M. Isolated cortical computations during delta waves support memory consolidation. Science 2019; 366: 377-81.

3. Miyamoto D, Hirai D, Murayama M. The roles of cortical slow waves in synaptic plasticity and memory consolidation. Front Neural Circuits 2017; 11: 92.

4. Girardeau G, Benchenane K, Wiener SI, Buzsaki G, Zugaro MB. Selective suppression of hippocampal ripples impairs spatial memory. Nat Neurosci 2009; 12: 1222-3.

5. Norimoto H, Makino K, Gao M, Shikano Y, Okamoto K, Ishikawa T, et al. Hippocampal ripples downregulate synapses. Science 2018; 359: 1524-7.

6. Davidson TJ, Kloosterman F, Wilson MA. Hippocampal replay of extended experience. Neuron 2009; 63: 497-507.

7. Foster DJ, Wilson MA. Reverse replay of behavioural sequences in hippocampal place cells during the awake state. Nature 2006; 440: 680-83.

8. Lee AK, Wilson MA. Memory of sequential experience in the hippocampus during slow wave sleep. Neuron 2002; 36: 1183-94.

9. Qin YL, McNaughton BL, Skaggs WE, Barnes CA. Memory reprocessing in corticocortical and hippocampocortical neuronal ensembles. Philos Trans R Soc Lond B Biol Sci 1997; 352: 1525-33. 
10. Khodagholy D, Gelinas JN, Buzsaki G. Learning-enhanced coupling between ripple oscillations in association cortices and hippocampus. Science 2017; 358: 369-72.

11. Wilber AA, Skelin I, Wu W, McNaughton BL. Laminar organization of encoding and memory reactivation in the parietal cortex. Neuron 2017; 95: 1406-19.e5.

12. Karimi Abadchi J, Nazari-Ahangarkolaee M, Gattas S, Bermudez-Contreras E, Luczak A, McNaughton BL, et al. Spatiotemporal patterns of neocortical activity around hippocampal sharp-wave ripples. Elife 2020; 9: e51972.

13. Logothetis NK, Eschenko O, Murayama Y, Augath M, Steudel T, Evrard HC, et al. Hippocampal-cortical interaction during periods of subcortical silence. Nature 2012; 491: 547-53.

14. Birba A, Hesse E, Sedeno L, Mikulan EP, Garcia MDC, Avalos J, et al. Enhanced working memory binding by direct electrical stimulation of the parietal cortex. Front Aging Neurosci 2017; 9: 178.

15. Catenoix H, Magnin M, Guenot M, Isnard J, Mauguiere F, Ryvlin P. Hippocampal-orbitofrontal connectivity in human: an electrical stimulation study. Clin Neurophysiol 2005; 116: 1779-84.

16. Scherf T, Angenstein F. Hippocampal CA3 activation alleviates fMRI-BOLD responses in the rat prefrontal cortex induced by electrical VTA stimulation. PLoS One 2017; 12: e0172926.

17. Funayama K, Minamisawa G, Matsumoto N, Ban H, Chan AW, Matsuki N, et al. Neocortical rebound depolarization enhances visual perception. PLoS Biol 2015; 13: e1002231.

18. Ishikawa D, Matsumoto N, Sakaguchi T, Matsuki N, Ikegaya Y. Operant conditioning of synaptic and spiking activity patterns in single hippocampal neurons. J Neurosci 2014; 34: 5044-53.

19. Matsumoto N, Okamoto K, Takagi Y, Ikegaya Y. 3-Hz subthreshold oscillations of CA2 neurons in vivo. Hippocampus 2016; 26: 1570-78.

20. Sato Y, Miyawaki T, Ouchi A, Noguchi A, Yamaguchi S, Ikegaya Y. Quick visualization of neurons in brain tissues using an optical clearing technique. Anat Sci Int 2019; 94: 199-208.

21. Epsztein J, Brecht M, Lee AK. Intracellular determinants of hippocampal CA1 place and silent cell activity in a novel environment. Neuron 2011; 70: 109-20.

22. Alexander AS, Nitz DA. Retrosplenial cortex maps the conjunction of internal and external spaces. Nat Neurosci 2015; 18: 1143-51.

23. Buzsaki G. Hippocampal sharp wave-ripple: a cognitive biomarker for episodic memory and planning. Hippocampus 2015; 25: 1073-188.

24. Joo HR, Frank LM. The hippocampal sharp wave-ripple in memory retrieval for immediate use and consolidation. Nat Rev Neurosci 2018; 19: 744-57.

25. Todorova R, Zugaro M. Hippocampal ripples as a mode of communication with cortical and subcortical areas. Hippocampus 2020; 30: 39-49.

26. Pagliardini S, Funk GD, Dickson CT. Breathing and brain state: urethane anesthesia as a model for natural sleep. Respir Physiol Neurobiol 2013; 188: 324-32.

27. Hulse BK, Moreaux LC, Lubenov EV, Siapas AG. Membrane potential dynamics of CA1 pyramidal neurons during hippocampal ripples in awake mice. Neuron 2016; 89: 800-13.

28. Calton JL, Taube JS. Where am I and how will I get there from here? A role for posterior parietal cortex in the integration of spatial information and route planning. Neurobiol Learn Mem 2009; 91: 186-96.

29. Whitlock JR, Sutherland RJ, Witter MP, Moser MB, Moser EI. Navigating from hippocampus to parietal cortex. Proc Natl Acad Sci U S A 2008; 105: 14755-62.

30. Roth FC, Beyer KM, Both M, Draguhn A, Egorov AV. Downstream effects of hippocampal sharp wave ripple oscillations on medial entorhinal cortex layer V neurons in vitro. Hippocampus 2016; 26: 1493-508.

31. Kerr KM, Agster KL, Furtak SC, Burwell RD. Functional neuroanatomy of the parahippocampal region: the lateral and medial entorhinal areas. Hippocampus 2007; 17: 697-708. 
32. LaChance PA, Todd TP, Taube JS. A sense of space in postrhinal cortex. Science 2019; 365: eaax4192.

33. Opalka AN, Huang WQ, Liu J, Liang H, Wang DV. Hippocampal ripple coordinates retrosplenial inhibitory neurons during slow-wave sleep. Cell Rep 2020; 30: 432-41.

34. Hill RA, Li AM, Grutzendler J. Lifelong cortical myelin plasticity and age-related degeneration in the live mammalian brain. Nat Neurosci 2018; 21: 683-95.

35. Sturrock RR. Myelination of the mouse corpus callosum. Neuropathol Appl Neurobiol 1980; 6: 415-20.

36. Vincze A, Mázló M, Seress L, Komoly S, Abrahám H. A correlative light and electron microscopic study of postnatal myelination in the murine corpus callosum. Int J Dev Neurosci 2008; 26: 575-84.

37. Baumann N, Pham-Dinh D. Biology of oligodendrocyte and myelin in the mammalian central nervous system. Physiol Rev 2001; 81: 871-927.

Open Access. This is an open-access article distributed under the terms of the Creative Commons Attribution-NonCommercial 4.0 International License (https://creativecommons.org/licenses/by-nc/4.0/), which permits unrestricted use, distribution, and reproduction in any medium for non-commercial purposes, provided the original author and source are credited, a link to the CC License is provided, and changes - if any - are indicated. 